\title{
Investigating the flyby scenario for the HD 141569 system
}

\author{
R. Reche, H. Beust, and J.-C. Augereau \\ Laboratoire d'Astrophysique de Grenoble, CNRS, Université Joseph Fourier, UMR 5571, 38400 Saint-Martin d'Hères, France \\ e-mail: Remy.Reche@obs.ujf-grenoble.fr
}

Received 19 June 2008 / Accepted 25 September 2008

\begin{abstract}
Context. HD 141569, a triple star system, has been intensively observed and studied because of its massive debris disk. Until recently, it was regarded as a gravitationally bound triple system but recent measurements of the HD 141569A radial velocity seem to invalidate this hypothesis. The flyby scenario therefore has to be further investigated to test its compatibility with the observed disk structures. Aims. We present a study of the flyby scenario for the HD 141569 system by considering 3 variants: a sole flyby, a flyby associated with one planet embedded in the disk and a flyby with two planets in the disk. We discuss the merits of each one to reproduce the scattered light observations of the disk.

Methods. We first use analytical calculations to reduce the parameter space of the 2 stellar companion orbit and then perform $N$-body numerical simulations of the flyby encounter using symplectic integration, taking into account the gravitational influence of the stars and the planets on massless test particles.

Results. The binary orbit is found to be almost fixed by the observational constraint on an edge-on plane with respect to the observers. If the binary had an influence on the disk structure, it should have a passing time at the periapsis between 5000 and 8000 years ago and a distance at periapsis between 600 and $900 \mathrm{AU}$. It also appears that the best scenario to reproduce the disk morphology is a flyby with 1 planet embedded in the disk. For a $2 M_{J}$ planet, its orbital eccentricity must be around 0.2 while for a $8 M_{J}$ planet, it must be below 0.1. In the two cases, its apoapsis is about $130 \mathrm{AU}$.

Conclusions. Although the global disk shape is reasonably well reproduced, some observed features cannot be explained by the present model and the likehood of the flyby event remains an issue for the scenario explored in this paper. Dynamically speaking, HD 141569 is still a puzzling system.
\end{abstract}

Key words. celestial mechanics - stars: planetary systems - methods: $N$-body simulations - stars: individual: HD 141569 methods: analytical

\section{Introduction}

HD 141569, a triple star system, has been intensively observed and studied because of its circumstellar dusty disk, imaged in scattered light in the visible and at near-infrared wavelengths. This system is located at $99 \pm 10 \mathrm{pc}$ and its age is estimated to be $5 \pm 3$ Myr (Weinberger et al. 2000; Merín et al. 2004). The disk is associated with the central star (B9.5 Ve) while the two other companions (M2 and M4) form a binary.

The resolved images of the disk, showing a complicated morphology, have generated much of discussion in the literature about the system dynamics (see Table 1 for a summary). First, this disk is in transition to a debris disk: although it is a dusty optically thin disk with a fractional disk luminosity of $L_{\text {disk }} / L_{*} \simeq 8.4 \times 10^{-3}$ (Sylvester et al. 1996), the gas mass is not negligible. It could in fact represent most of the mass according to Jonkheid et al. (2006) $\left(M_{\text {gas }}=80 M_{\oplus}, M_{\text {dust }}=2.2 M_{\oplus}\right.$ for grains with radius between $1 \mu \mathrm{m}$ and $1 \mathrm{~cm}$ ), although the spatial distribution of the gas and of the dust may differ. The influence of the gas on the dust dynamics therefore has been taken into account by Ardila et al. (2005), but other authors consider only classical $N$-body simulations without gas (Augereau \& Papaloizou 2004; Wyatt 2005). Quillen et al. (2005), on the other hand, only considered the gas in their simulations.

The dynamical status of the external binary is also questionable: in order to reproduce the external structures of the disk, previous studies have either considered the case of gravitationally bound companions (Augereau \& Papaloizou 2004; Quillen et al. 2005) or the case of a flyby (Ardila et al. 2005). The internal disk structures might be explained by the gravitational perturbation of an unseen planet at large distance, around $250 \mathrm{AU}$ (Wyatt 2005) but also by alternative mechanisms, such as dust migration in a gaseous disk (Takeuchi \& Artymowicz 2001). The latter scenario is successful in producing annular structures, but cannot account for the observed non-axisymmetric nature of these features. The combined effect of a planet and external perturbers has only been considered in one study, without successfully reproducing all the structures (Ardila et al. 2005).

New millimeter observations of the gas disk (Dent et al. (2005) and Augereau et al., in prep.), which give better constraints on the radial velocity of the primary star, show that the differential velocity between the binary and the central star $\left(5.8 \pm 0.3 \mathrm{~km} \mathrm{~s}^{-1}\right)$ is higher than the system escape velocity $\left(2.6 \mathrm{~km} \mathrm{~s}^{-1}\right)$ so that the flyby scenario seems to be the most plausible. Therefore, in this paper we propose to extensively study the flyby scenario, as it is the least studied up to now. In Sect. 2 we summarise the available observations of the disk and the star astrometry constraints. In order to best reproduce the observations, we discuss 3 different scenarios (Fig. 1): a sole flyby (Sect. 3), a flyby with 1 planet embedded in the disk (Sect. 4) and a flyby with 2 planets embedded in the disk (Sect. 5). In Sect. 6 , 
Table 1. Summary of recent papers on the dynamical modelling of the HD 141569 system.

\begin{tabular}{ccccc}
\hline \hline Authors & Modelled structures & External companions & Planet & Notes \\
\hline Takeuchi \& Artymowicz (2001) & gap \& two rings & No & No & Dust migration due to gas friction \\
Augereau \& Papaloizou (2004) & outer ring & bound & No & $N$-body gravitational code \\
Quillen et al. (2005) & outer ring & bound & No & 2D hydrodynamics code \\
Ardila et al. (2005) & spiral arms \& inner depletion & flyby & Yes & $N$-body code, SPH code, collisions \\
Wyatt (2005) & gap \& outer ring & No & Yes & $N$-body gravitation code \\
\hline
\end{tabular}
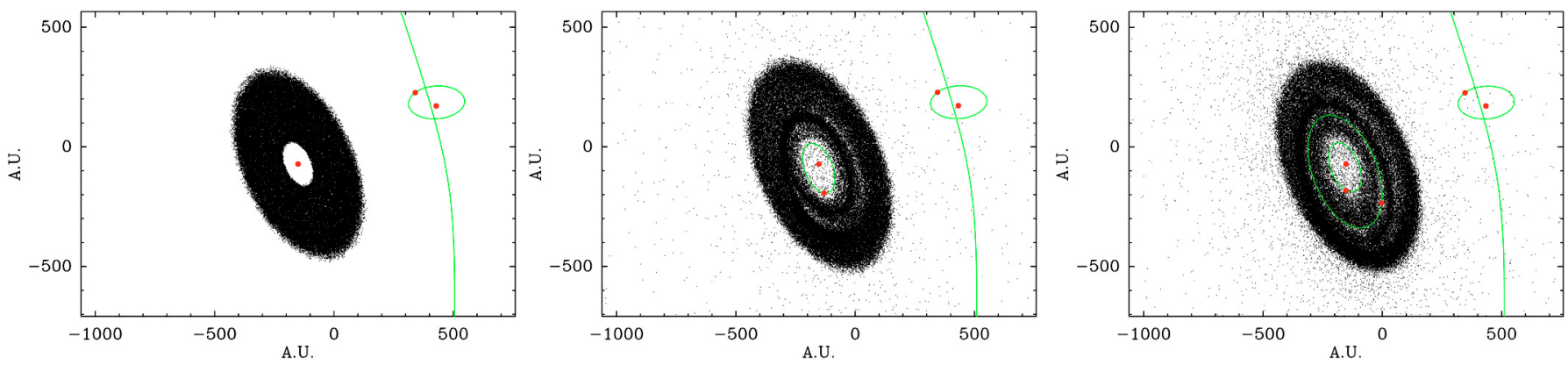

Fig. 1. Illustrations of the 3 scenarios studied in this paper: a sole flyby (left panel), a flyby +1 planet (middle panel) and a flyby +2 planets (right panel). The stars and planet locations are represented by large red points, and the various orbits by a thin green line. (This figure is available in color in electronic form.)

we compare our approach to previous studies and discuss in particular the likelihood of this scenario. We finally summarise and conclude in Sect. 7.

\section{Available observations}

\subsection{Overview of the disk shape}

The motivation for our study is to reproduce the dust disk structures that have been observed in scattered light. According to the visible, near-infrared and mid-infrared observations from Augereau et al. (1999), Weinberger et al. (1999), Mouillet et al. (2001), Marsh et al. (2002) and Clampin et al. (2003) the following facts appear:

- the main structure is the two ring shape of the disk. The two annuli peak at $\sim 200 \mathrm{AU}$ and $\sim 325 \mathrm{AU}$ from the star. The two bright rings show out of phase brightness asymmetries of up to factors of 2.5-3 for the outer ring in the visible. The outer ring moreover shows a tightly-wound spiral structure. The two annuli are separated by a darker ring or "gap", which is radially wide compared to the two annuli;

- an extended diffuse emission associated with a faint spiral arm is present in the North-East of the disk and is detected at to more than $600 \mathrm{AU}$. According to the millimeter maps and using the hypothesis of forward scattering, this spiral would be a trailing structure with respect to the disk rotation. Another spiral arm is possibly observed, pointing toward the companions;

- the disk brightness sharply decreases between 200 and $150 \mathrm{AU}$ from the central star until the background level is reached. This behaviour is suggestive of a strong depletion of dust inside $150 \mathrm{AU}$.

\subsection{Radial velocities and astrometry}

Weinberger et al. (2000) have measured the radial velocities of the HD 141569B and C stars, respectively $-1.5 \pm 0.6 \mathrm{~km} \mathrm{~s}^{-1}$ and $-2.4 \pm 0.7 \mathrm{~km} \mathrm{~s}^{-1}$, by cross-correlating their spectra to that of standard stars. This method was not suitable for HD 141569A due to its lack of lines for comparison with the radial velocity standard. Weinberger et al. (2000) also summarise the astrometry of the 3 stars from 1938 to 1999.

Augereau et al. (in prep.) and Dent et al. (2005) have measured the double-peaked CO $J=2-1$ spectral profile of the disk around HD 141569A. It is thus possible to deduce the radial velocity of this star from the $\mathrm{CO}$ line and to obtain a far smaller uncertainty on this measure. To a first approximation, assuming a symmetric emission in the blue-shifted and red-shifted parts of the disk, the radial heliocentric velocity of HD 141569A is found to be $-7.6 \pm 0.3 \mathrm{~km} \mathrm{~s}^{-1}$ (mean velocity in the line). Supposing that the star masses are 2.5, 0.5 and $0.25 M_{\odot}$ for A-C respectively (Weinberger et al. 2000) and that the distance between HD 141569A and the center of mass of the B-C binary is at least $800 \mathrm{AU}$ (projected distance, Weinberger et al. (2000)), the maximum escape velocity is $2.6 \mathrm{~km} \mathrm{~s}^{-1}$. As the measured velocity is far above this limit, the two $\mathrm{M}$ star companions are not bound to HD 141569A. In the context of dynamical perturbation of the disk by the companions, the flyby scenario seems therefore to be the most plausible.

\section{The flyby scenario}

\subsection{Kinematic constraints}

We define a Cartesian coordinate system $(X, Y, Z)$ where $X$ points toward the north, $Y$ toward the east and $Z$ toward the Earth and where the origin is HD 141569A. In this referential frame, the position of the center of mass of the B-C binary will be described by the coordinates $\left(X_{b}, Y_{b}, Z_{b}\right)$ and its velocity by $V_{b_{x}}, V_{b_{y}}, V_{b_{z}}$. We already know the present $X_{b}, Y_{b}$ (right ascension and declination) and $V_{b_{z}}$ (radial velocity). But the hyperbolic orbit of the binary around the primary is also defined by the 6 orbital elements $(q, e, i, \omega, \Omega, u)$ where $q$ is the pericenter distance, $e$ the eccentricity, $i$ the inclination with respect to the sky plane $(O X Y)$, $\omega$ the periapsis argument, $\Omega$ the longitude of the ascending node and $u$ the eccentric anomaly at the observing time. The transformation from Cartesian coordinates to orbital elements, or the inverse transformation, is defined by a system of 6 nonlinear equations from a classical Keplerian formalism. It is thus 
possible to look for orbital element set verifying the 3 observables $\left(X_{b}, Y_{b}, V z_{b}\right)$. But with 6 unknowns and only 3 constraints the equation system is underconstrained. One therefore has to consider 3 orbital elements as free parameters in order to solve the system for the 3 other orbital elements. This system is defined by the following equations, for hyperbolic orbit:

$$
\begin{aligned}
X_{b} \cos \Omega-Y_{b} \sin \Omega= & a \cos i\left(\sqrt{e^{2}-1} \cos \omega \sinh u\right. \\
& +(e-\cosh u) \sin \omega) . \\
X_{b} \sin \Omega+Y_{b} \cos \Omega= & a\left(-\sqrt{e^{2}-1} \sin \omega \sinh u\right. \\
& +(e-\cosh u) \cos \omega) . \\
V_{b_{z}} \sqrt{a}(e \cosh u-1)= & \sqrt{G M} \sin i(\sinh u \sin \omega \\
& \left.-\sqrt{e^{2}-1} \cosh u \cos \omega\right) .
\end{aligned}
$$

where $G$ is the gravitational constant and $M$ the sum of the 3 star masses. For this study, we choose to consider $(i, \Omega, u)$ as free parameters and $(q, e, \omega)$ as unknowns. Once the 3 parameters $(i, \Omega, u)$ are fixed to an arbitrary set of values, the non-linear system generally admits none or one solution for the triplet $(q, e, \omega)$. For our problem, many triplets of the parameters $(i, \Omega, u)$ give a solution: for each $(i, u)$ pair, at least one value of $\Omega$ gives a valid solution for $(q, e, \omega)$. The results are thus not significant because all the scenarios can be compatible with the observations: orbital plane perpendicular to the line of sight, or instead very inclined orbits, more or less eccentric hyperbolic orbits ...

However, another constraint exists, not yet used in our method: the observed proper motion of the stars between 1938 and 1998 (Weinberger et al. 2000), and the associated velocity $V_{\text {sky }}=1.1 \pm 0.7 \mathrm{~km} \mathrm{~s}^{-1}$. The uncertainty on these observables is quite large and we consider it as an upper limit: all the solutions that predict a proper motion less than twice the observed proper motion are valid:

$$
\sqrt{V_{b_{x}}^{2}+V_{b_{y}}^{2}}<2 V_{\text {sky }}
$$

This is enough to reduce significantly the number of solutions, as showed in Fig. 2. On this plot, the two axes are the eccentric anomaly $u$ and the inclination $i$, while the colors correspond to the density of valid solutions (i.e. fulfilling the relation 1 to 4 ) for the longitude of the ascending node $\Omega$ in the range $[0,2 \pi]$. The parameter space region where the valid solutions exist is indeed very much reduced by using the proper motion constraint, as we find with an inclination $i$ between $75^{\circ}$ and $105^{\circ}$ and an eccentric anomaly $u$ between -5 and 5 radians. The fourth constraint has therefore eliminated all the solutions with lower inclination and larger proper motion.

The disk inclination is $55^{\circ} \pm 1.2^{\circ}$ from pole-on (Mouillet et al. 2001), or rather $180^{\circ}-55^{\circ}$ in order to have the disk rotation (Goto et al. 2006), consistent with our convention (i.e. $i<90^{\circ}$ is an anticlockwise orbit on the sky). The binary orbits with an inclination below (resp. above) $90^{\circ}$ are thus retrograde (resp. prograde) with respect to the disk rotation. The relative inclination $i_{\text {rel }}$ between the orbital plane of the binary and that of the disk can be calculated by the following equation:

$\cos i_{\text {rel }}=\cos i \cos i_{\text {disk }}+\sin i \sin i_{\text {disk }} \cos \left(\Omega-\Omega_{\text {disk }}\right)$

with $\Omega_{\text {disk }}$, the position angle of the disk $\left(357^{\circ} \pm 2^{\circ}\right)$ and $i, \Omega$ orbital elements of the binary orbit.

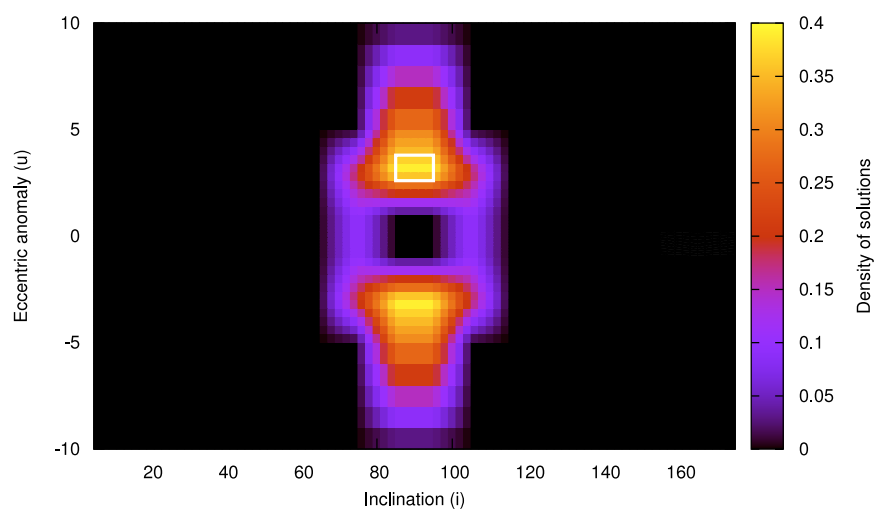

Fig. 2. Density of valid orbital configurations in the parameter space $(i, u, \Omega)$. The two axes are the eccentric anomaly $u$ and the inclination $i$, while the colors correspond to the density of the valid solutions for the longitude of the ascending node $\Omega$ in the range $[0,2 \pi]$. The small white box shows the final valid parameter space when the disk geometry constraints are also taken in account (Sect. 3.2). (This figure is available in color in electronic form.)

\subsection{Disk geometry constraints}

At this point, only the kinematic constraints have been used to calculate the valid orbital configurations for the binary. However, the encounter geometry is also constrained by the observed structures in the disk if one assumes that the current disk shape is a consequence of the gravitational perturbations of the disk by the binary. The comparison study of numerical simulations of the flyby scenario to the observations therefore reduces even more the range of valid orbital configurations, as detailed below.

\subsubsection{Numerical model}

We consider in our simulations a hierarchical system consisting of a central star associated with a debris disk and an external binary. The trajectory of this binary can be a bound orbit around the primary star or a flyby (hyperbolic orbit). We address the case of a disk consisting of large particles (planetesimals), which are insensitive to pressure forces (radiation, stellar wind or gas pressure). Importantly, we also do not take into account the gravitational interactions between planetesimals as they are negligible, nor do we consider mutual collisions. Dynamically speaking, the planetesimals are thus considered as test particles. In Sects. 4 and 5, one or several planets will be added to the simulations, orbiting the central star in the orbital plane of the disk. The central star is assumed to have a mass of $2.5 M_{\odot}$ and the low mass companions of $0.5 M_{\odot}$ and $0.25 M_{\odot}$ (Weinberger et al. 2000). The initial disk consists of 100000 test particles with a surface density distribution proportional to $r^{-1}$.

To perform our simulations we use the symplectic package HJS (Beust 2003), a SWIFT variant (Wisdom \& Holman 1991; Levison \& Duncan 1994) for hierarchical systems. It allows us to integrate accurately and rapidly the motion of the disk particles, the relative motion of the two companions and their orbit around the central star, although they are on different timescales. Numerous scenarios can be therefore tested and studied in a reasonable CPU time.

\subsubsection{Results}

Among the solutions consistent with the kinematic constraints (Sect. 3.1), the periapsis of the orbit, $d_{0}$ (i.e. the closest 

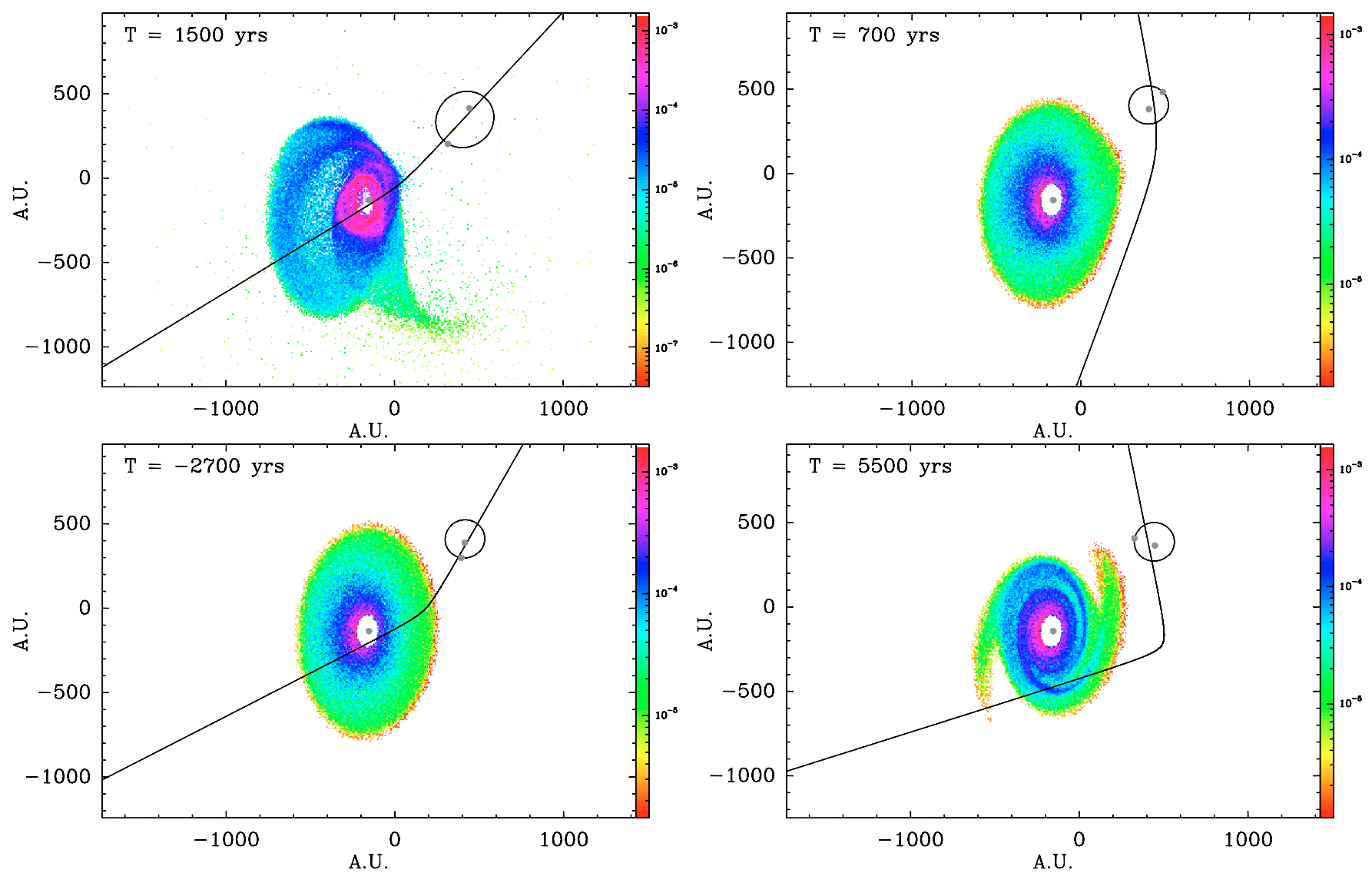

Fig. 3. Examples of parameters for the flyby scenario inconsistent with the observations: the periapsis is too close to the disk, at 170 AU (top left panel), the crossing time at periapsis is too recent, 700 years (top right), the binary is still not yet at periapsis (bottom left) and the disk is too large (bottom right). These figures plot the intensity, in logarithmic scale, of scattered light by the planetesimal spatial distribution obtained with our simulations. The scattering anisotropic factor is 0.2 . The time is relative to the crossing time at periapsis, $t_{0}$.

approach) can range between 10 and several thousands of AU. If the flyby is however too close to the star, the disk can be totally dissipated or, at least, can show a spiral structure which strongly differs from the observations (Fig. 3 top left panel). We numerically explored a broad range of $d_{0}$ values consistent with the kinematic constraints of Sect. 3.1. According to the simulations, we find that the distance at periapsis, $d_{0}$, must be above about $600 \mathrm{AU}$. An other useful constraint is the time span between the periapsis passage and the present time, $T=t-t_{0}$. If $T$ is too small compared to the Keplerian period at the outer disk edge, the spiral structure does not have the time to develop (Fig. 3 top right panel). The simulations give an estimate of at least 5000 years before the present time for the periapsis passage. All the well defined structures in the disk develop after the periapsis passage, never before, which eliminates all the configurations with $u<0$, because the binary is still too far to generate any perturbations in the disk (Fig. 3 bottom left panel).

Few orbital configurations finally verify the system given by the Eqs. (1) to (4) plus the additional constraints $d_{0}>600 \mathrm{AU}$ and $T=t-t_{0}>5000$ yrs. Valid solutions are only possible for $i$ between $85^{\circ}$ and $95^{\circ}$ and $u \approx 3$ and share the following properties:

$5000 \mathrm{yrs}<t-t_{0}<8000 \mathrm{yrs}$

$600 \mathrm{AU}<d_{0}<900 \mathrm{AU}$

$e \approx 10$.
From Eq. (5), it is also possible to determinate that:

$35^{\circ}<i_{\text {rel }}<145^{\circ}$

Considering these solutions, the binary has at present already reached its velocity at infinity. Within this range of solutions, the initial size of the disk can also be better constrained by our simulations in the range between 400-450 AU. If it is larger (Fig. 3 bottom right panel), the flyby produces large spiral arms which are too extended while if the disk size is smaller, the disk does not fit the observed size. We do not address here the question of the origin of the disk size, nor the shape of the external disk edge. But collisional evolution of the disk (Thébault \& Wu 2008) or gravitationnal truncation by the binary in a past bound state of the system (see Sect. 6.1) can both explain this.

Overall, this scenario gives satisfactory results for the global shape of the disk and reduces significantly the range of possible orbital configurations for the companions. This is illustrated in Fig. 4 which shows a disk of correct size with an outer trailing spiral. The inner disk structure is nevertheless not properly reproduced and the next step is thus to reproduce more accurately the two ring-like structure at distances of about 200 and $325 \mathrm{AU}$ from the star (Mouillet et al. 2001; Clampin et al. 2003). It appears however that none of the valid solutions for the flyby is able to generate the two rings well. In the case of a retrograde orbit $\left(85^{\circ} \leq i<90^{\circ}\right)$, the flyby generates a clear two armed spiral structure (Fig. 4, left panel). In the case of a prograde orbit $\left(90^{\circ}<i \leq 95^{\circ}\right)$, as studied for instance by Pfalzner (2003), the two arms are more wound and more extended but the model is 

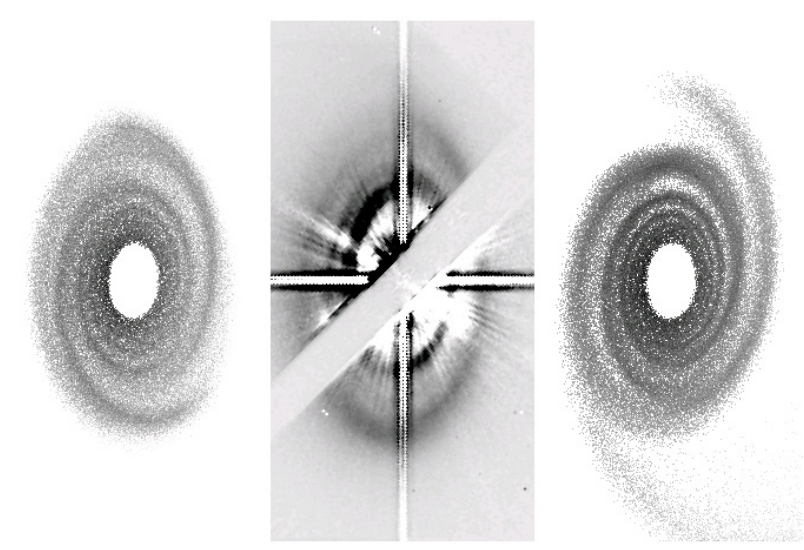

Fig. 4. Best solutions for the flyby scenario on a retrograde orbit (left panel) and on a prograde orbit (right panel), compared to the observations from Mouillet et al. (2001) (middle panel). A scattering anisotropic factor $g$ of 0.2 is assumed to mimic real scattered light observations.

still too different from the observations (Fig. 4, right panel). In the two cases, the flyby does not perturb the inner part of the disk, below 200 AU. It thus cannot explain the inner ring, nor the observed depletion around $150 \mathrm{AU}$. This means that we need to add some other hypothesis to the model.

\section{The flyby + planet scenario}

We can conclude from Sect. 3 that the presence of the two star companions is not enough to explain the observed disk properties summarised in Sect. 2, especially the double-ring structure. The flyby can, at least, constrain the initial disk size between 400 and 450 AU but it is necessary to use other hypothese to improve the model and to better fit the disk geometry. As the observations show a strong depletion in the dust surface density below $150 \mathrm{AU}$, a plausible hypothesis is the presence of an unseen planet which generates this gap.

In this section, we consider the case where we add a planet within $150 \mathrm{AU}$ in our numerical models. The planet is supposed to be coplanar with the disk and its main parameters are its mass, its orbital semi-major axis and its orbital eccentricity. The simulations take into account the mutual gravitational interactions between the planet and the 3 stars, as well as the gravitational perturbation of the disk by the planet but not the feedback of the disk on the planet: the planetesimals are still considered as test particles. The system consisting of the central star, the disk and the planet is first evolved for 5 Myr (the assumed age of the system), then the two star companions are introduced to simulate the flyby with one of the valid set of orbital parameters found in Sect. 3.

\subsection{The inner ring}

To constrain the planet parameters, we compare the simulations to the disk optical thickness derived from Marsh et al. (2002). The authors combined their own observations in the mid-infrared and the Weinberger et al. (1999) observations in the near-infrared (Fig. 5) to estimate the radial profiles of the vertical dust optical depth at $1.1 \mu \mathrm{m}$. The observations in the mid-infrared cover the inner region of the disk up to $130 \mathrm{AU}$ while the observations in the near-infrared cover the region between 130 and 500 AU. The radial profile of the optical depth at $1.1 \mu \mathrm{m}$ is deduced for the mid-infrared data from the optical depth at $12.5 \mu \mathrm{m}$ and assuming the opacity scales inversely with wavelength.

The profiles show a ring which peaks around $200 \mathrm{AU}$, a depletion at $250 \mathrm{AU}$ and an even more depleted region below $150 \mathrm{AU}$. In the following we use these data to constrain the mass and the orbital elements of the planet, by trying to reproduce some key characteristics of the azimuthal averaged radial density profile of the dust:

- a peak between 190 and $210 \mathrm{AU}$;

- a ratio $\tau_{\text {peak }} / \tau_{250}$ of about 1.3 between the peak surface density at $\sim 200 \mathrm{AU}$ and the depletion at $\sim 250 \mathrm{AU}$;

- a ratio $\tau_{\text {peak }} / \tau_{150}$ of about 2.7 between the peak at $\sim 200 \mathrm{AU}$ and the inner depleted region at 150 AU. The uncertainty on this ratio is nevertheless rather large because of the large uncertainties in the optical depth between 100 and 150 AU.

The "1 planet + flyby" scenario is tested for 3 different planet masses: $0.2,2$ and $8 M_{J}$. For each planet mass, 3 apoapsis are considered (130, 150 and $180 \mathrm{AU})$ with 4 different values of the eccentricity: $0,0.05,0.1$ and 0.2 . This gives a total of 36 simulations and their results are summarised in Tables 2-4. The peak position (Table 2) indicates the position of the largest overdensity if several are present. $\tau_{\text {peak }} / \tau_{150}$ is defined as the ratio between the dust density at the peak position and the maximum density between 100 and $150 \mathrm{AU} . \tau_{\text {peak }} / \tau_{250}$ is defined as the ratio between the dust density at the peak position and the density around $250 \mathrm{AU}$.

According to these simulations, a $0.2 M_{J}$ planet cannot reproduce the observational data: it does not clear out the inner part of the disk enough. For higher mass planets, the best solution is obtained for an apoapsis of 130 AU. For larger values of apoapsis, the peak position is shifted toward too large distance from the star, or the planet can even prevent the formation of an over-density. For a $2 M_{J}$ planet, the best fits are obtained for eccentricities around 0.2 , while for a $8 M_{J}$ planet, it must be below 0.1 . The best solution between these two planets is for a $2 M_{J}$ planet.

With an initial surface density distribution proportional to $r^{-1}$, Fig. 5 shows that there is a lack of particles in the outer part of the disk compared to the optical depth profile at $\lambda=1.1 \mu \mathrm{m}$. We therefore adjust the initial surface density to obtain the same maximum at the outer peak location, near 330 AU. We find that the best fit is obtained for an initial surface density distribution proportional to $r^{-0.5}$ and that the outer peak position in our simulation is consistent with the observations. However, there is still a lack of particles between the two rings and outside the outer ring. This might reflect a limitation of our model as we only model the dynamics of planetesimals. One can expect that smaller dust particles, sensitive to radiation pressure and generated by collisions, will migrate outside the rings and flatten the profiles, hence better reproducing the optical depth profile.

\subsection{The global structure}

The ratio between the two peaks in the optical depth profile depends on the observing wavelength, as the optical depth at the outer peak position is larger than the optical depth at the inner peak position at $0.5 \mu \mathrm{m}$ (Ardila et al. 2005), while it is the opposite in Fig. $5(\lambda=1.1 \mu \mathrm{m})$. Our constraint on the initial surface density distribution should therefore be taken with care, but our model is nevertheless very successful in predicting the position 
Table 2. Summary of the results for the peak position (in AU) in the planetesimal density profile, for all the simulations, as a function of the planet mass, eccentricity and apoapsis. "_" Indicates the simulations where no peak is observable or where the peak is below 170 AU. $\dagger$ Indicates the simulations where multiple peaks appear. In this case, the largest peak is used to calculate the peak position, $\tau_{\text {peak }} / \tau_{150}$ and $\tau_{\text {peak }} / \tau_{250}$. The numbers in boldface correspond to the best solutions.

\begin{tabular}{|c|c|c|c|c|c|c|c|c|c|c|c|c|}
\hline Planet mass & \multicolumn{4}{|c|}{$\overline{0.2 M_{J}}$} & \multicolumn{4}{|c|}{$2 M_{J}$} & \multicolumn{4}{|c|}{$8 M_{J}$} \\
\hline Planet ecc. & 0 & 0.05 & 0.1 & 0.2 & 0 & 0.05 & 0.1 & 0.2 & 0 & 0.05 & 0.1 & 0.2 \\
\hline apoapsis of $180 \mathrm{AU}$ & 210 & 210 & 210 & - & - & 230 & $\overline{-}$ & - & $\overline{-}$ & - & $\overline{-}$ & - \\
\hline apoapsis of $150 \mathrm{AU}$ & $220 \dagger$ & - & - & 230 & - & $210 \dagger$ & 230 & 240 & - & - & - & - \\
\hline apoapsis of $130 \mathrm{AU}$ & - & - & - & 220 & - & $220 \dagger$ & - & 210 & 220 & 220 & 230 & 220 \\
\hline apoapsis of $120 \mathrm{AU}$ & - & - & - & - & - & - & 200 & 190 & 220 & 220 & 230 & 230 \\
\hline
\end{tabular}

Table 3. Same as Table 2, but for the ratio $\tau_{\text {peak }} / \tau_{150}$ between the density at peak position and the maximum density between 100 and 150 AU.

\begin{tabular}{|c|c|c|c|c|c|c|c|c|c|c|c|c|}
\hline 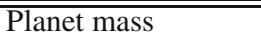 & \multicolumn{4}{|c|}{$0.2 \underbrace{M_{J}}_{J}$} & \multicolumn{4}{|c|}{$2 M_{J}$} & \multicolumn{4}{|c|}{$8 \underbrace{M_{J}}$} \\
\hline Planet ecc. & 0 & 0.05 & 0.1 & 0.2 & 0 & 0.05 & 0.1 & 0.2 & 0 & 0.05 & 0.1 & 0.2 \\
\hline apoapsis of $180 \mathrm{AU}$ & 0.5 & 0.5 & 0.5 & - & - & - & 0.4 & - & - & - & - & - \\
\hline apoapsis of $150 \mathrm{AU}$ & $0.5 \dagger$ & - & - & 0.8 & - & $0.6 \dagger$ & 1.6 & 3.0 & - & - & - & - \\
\hline apoapsis of $130 \mathrm{AU}$ & - & - & - & 1.1 & - & $2.4 \dagger$ & - & 2.3 & 3.0 & 5.0 & 6.0 & 8.0 \\
\hline apoapsis of $120 \mathrm{AU}$ & - & - & - & - & - & - & 1.3 & 2.1 & 2.7 & 4.7 & 4.2 & 4.5 \\
\hline
\end{tabular}

Table 4. Same as in Table 2, but for the ratio $\tau_{\text {peak }} / \tau_{250}$ between the density at peak position and the density in the gap around 250 AU.

\begin{tabular}{|c|c|c|c|c|c|c|c|c|c|c|c|c|}
\hline \multirow{2}{*}{$\begin{array}{l}\text { Planet mass } \\
\text { Planet ecc. }\end{array}$} & \multicolumn{4}{|c|}{$\overline{0.2} M_{J}$} & \multicolumn{4}{|c|}{$2 M_{J}$} & \multicolumn{4}{|c|}{$8 \underbrace{M_{J}}$} \\
\hline & 0 & 0.05 & 0.1 & 0.2 & 0 & 0.05 & 0.1 & 0.2 & 0 & 0.05 & 0.1 & 0.2 \\
\hline apoapsis of $180 \mathrm{AU}$ & 1.5 & 1.7 & 1.3 & - & - & - & 1.1 & - & - & - & - & - \\
\hline apoapsis of $150 \mathrm{AU}$ & $1.7 \dagger$ & - & - & 1.8 & - & $1.4 \dagger$ & 1.5 & 2.0 & - & - & - & - \\
\hline apoapsis of $130 \mathrm{AU}$ & - & - & - & 2.0 & - & $1.7 \dagger$ & - & 2.0 & 1.9 & 1.9 & 1.7 & 1.3 \\
\hline apoapsis of $120 \mathrm{AU}$ & - & - & - & - & - & - & 2.2 & 2.1 & 1.8 & 1.7 & 1.8 & 1.4 \\
\hline
\end{tabular}

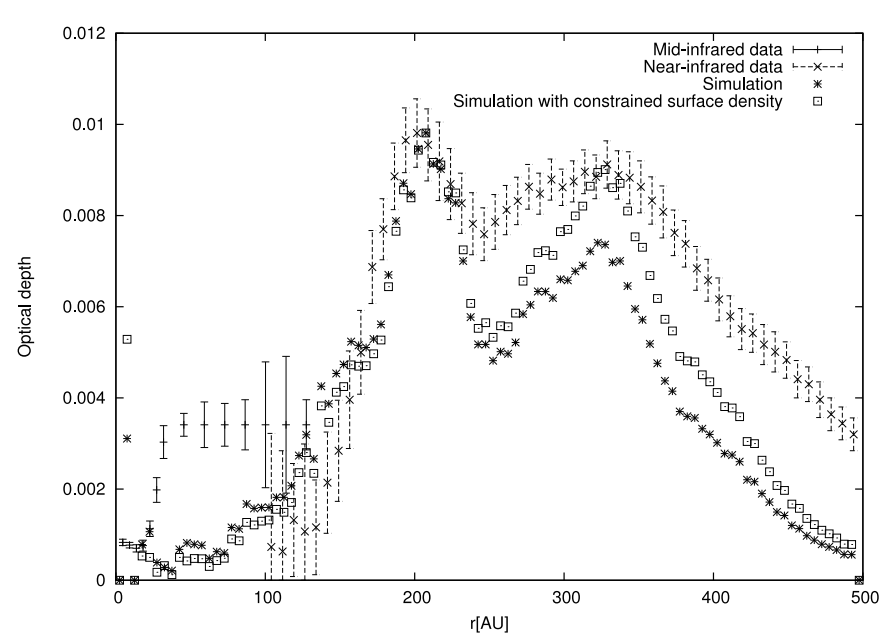

Fig. 5. Estimated profiles of dust optical depth (normal to the disk plane) by combining mid-infrared data (Marsh et al. 2002) and near-infrared data (Weinberger et al. 1999), compared to the simulation output of the best scenario, a $2 M_{J}$ planet with an eccentricity of 0.2 .

of the outer peak in the optical depth profile. The planet parameters are strongly constrained by the inner ring, but our simulations show that the planet also perturbs the outer parts of the disk, as shown in Figs. 5 and 6.

In less than $5 \mathrm{Myr}$, the planet has time enough to develop a strong, one-armed, anticlockwise wound spiral, due to the differential precession rate of the planetesimal orbits as explained by Augereau \& Papaloizou (2004). The flyby thus does not occur with an initially smooth disk and this has strong consequences for the final shape of the structures produced by the flyby. For instance, in the case where the binary is on a retrograde orbit, we previously obtained a regular two armed spiral. But now, the planet not only produces the inner ring, but can also remove, or at least perturb, a significant fraction of one of the two spiral arms. As shown in Fig. 6, the disk morphology between 200 and 300 AU depends slightly on the direction of the planet periapsis with respect to the direction of the binary periapsis, but the differences remain too small to constrain the orientation of the planet orbit. The results are thus not perfect because the simulations still do not exactly reproduce the outer ring but they clearly show that we need a complete description of the system to properly account for it. It is not satisfactory to consider solely the external companions or the inner planet. Combining both, we clearly achieve a much better fit to the observations.

\section{The flyby +2 planets scenario}

As the previous scenario is still not fully satisfactory because it still does not generate two rings clearly separated by a large gap, we have also investigated an alternative with two planets in the disk. Wyatt (2005) considered the case of a single planet at about $250 \mathrm{AU}$ to explain the outer ring but without considering the perturbation due to the companion stars. We therefore first investigate the consequence of both the companions and the planet on the disk geometry. Using the solutions found in Sect. 3, it appears that adding the gravitational influence of the two companion stars does not improve the structure generated by the external planet but, on the contrary, disrupts them because the planetesimal orbits are too destabilised by the perturbations of the planet and the two companions. For this scenario, the constraints used in Sect. 3.2 are no longer valid and the correct orbital elements for the binary orbit are those which prevent interactions between 

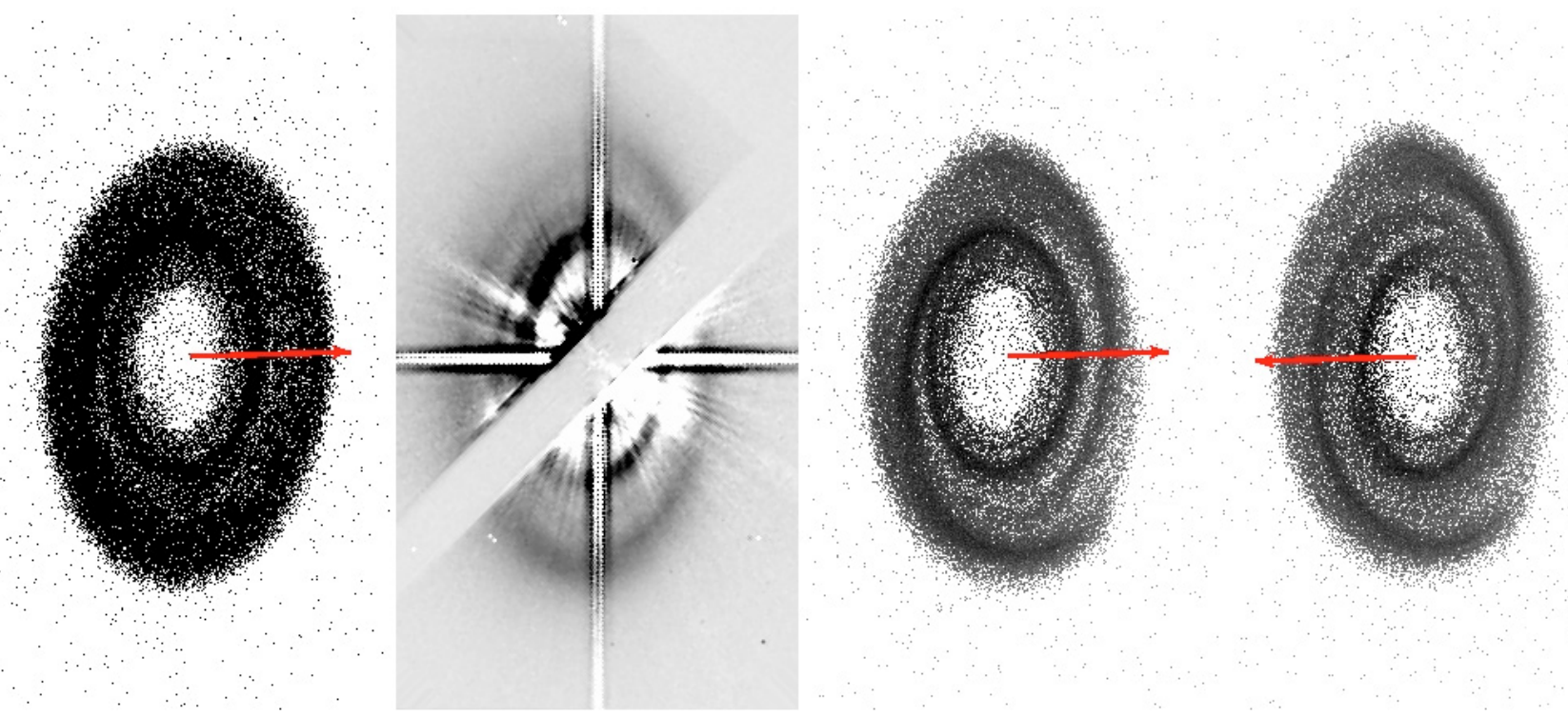

Fig. 6. Same as in Fig. 4, but for the best solution for the flyby + planet scenario in the case of a retrograde orbit of the binary (third panel). The red arrow indicates the direction of the planet periapsis. The planet mass is equal to $2 M_{J}$, its apoapsis is $130 \mathrm{AU}$ and its eccentricity 0.2 . The first panel show the disk before the flyby but after $5 \mathrm{Myr}$ of gravitational perturbation by the planet. The fourth panel shows an alternative configuration where the initial direction of the planet periapsis is turned by $180^{\circ}$.

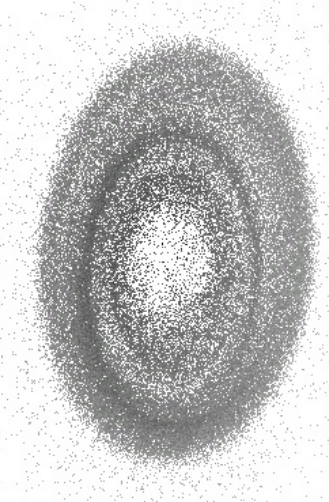

Fig. 7. Same as in Fig. 4 , but for the flyby +2 planets scenario. The inner planet mass is equal to $2 M_{J}$, it apoapsis is $130 \mathrm{AU}$ and its eccentricity 0.2 . The outer planet mass is $0.2 M_{J}$, it apoapsis is $250 \mathrm{AU}$ and its eccentricity 0.05 .

the disk and the two M stars: they still do not reach the periapsis location $(u<0)$, or the periapsis itself is located at more than 1500 AU from the primary star.

Such a scenario cannot, however, reproduce the inner depletion below $150 \mathrm{AU}$; another planet is thus necessary,as in the previous section. According to Wyatt (2005), two scenarios can be considered: a $0.2 M_{J}$ planet evolving over $5 \mathrm{Myr}$ on an orbit with a semi-major axis of $250 \mathrm{AU}$ and an eccentricity of 0.05 , and a $2 M_{J}$ planet perturbing the disk over $0.5 \mathrm{Myr}$ and on the same orbit. We add thus another planet in the internal part of the disk, following the best solutions found in the previous section, namely a $2 M_{J}$ planet on an orbit with a semi-major axis of $110 \mathrm{AU}$ and an eccentricity of 0.2 or a $8 M_{J}$ planet on an orbit with a semi-major axis of $115 \mathrm{AU}$ and an eccentricity of 0.05 (Fig. 7).

As in Sect. 4, the estimated radial profiles of the dust optical depth obtained with these simulations are compared to the observed optical depth at $1.1 \mu \mathrm{m}$ (Fig. 5). Two major pieces of information are therefore given by this method. First, and independently of the mass of the inner planet, a $2 M_{J}$ at $250 \mathrm{AU}$ generates a too deep gap in the disk compared to the observations (scenario B and C of Fig. 8). Contrary to scenario "flyby + 1 planet", one cannot expect that the optical depth profile would be flattened by smaller dust particles sensitive to radiation pressure because the particles will be quickly removed from the gap, due to close encounter with this planet orbiting at $250 \mathrm{AU}$. Wyatt (2005) constrains the planet parameters considering the location and the winding of the spiral but there is a degeneracy between the planet mass and the time the planet is orbiting around the star. If one adds the constraints given by the observations on the depth of the gap around $250 \mathrm{AU}$, only the scenarios with smaller planet masses $\left(0.2 M_{J}\right)$ orbiting for a long time $(5 \mathrm{Myr})$ are satisfactory (scenario A and D of Fig. 8)). Second, the simulations show that it is difficult to obtain an inner ring massive enough between the two planets at around 200 AU. Due to the perturbations of these two planets, many planetesimals are ejected from the disk. We have therefore tried other simulations with a smaller mass $\left(0.2 M_{J}\right.$, scenario D) for the internal planet in order to less perturb the disk. However, the results are still not satisfactory because the peak position is not at the correct location (150 AU instead of $200 \mathrm{AU}$ ) and because, with a low mass planet, there are too many planetesimals below $100 \mathrm{AU}$.

A planet located at $250 \mathrm{AU}$ from the primary star is a interesting alternative to the companions in order to explain the outer ring without the gravitational perturbation of the 2 stellar companions, but our work shows that combining it with another planet around $120 \mathrm{AU}$ to explain the inner ring and the dust depletion within $150 \mathrm{AU}$ does not provide satisfactory results. Even if it is still not perfect, the scenario "flyby +1 planet" 


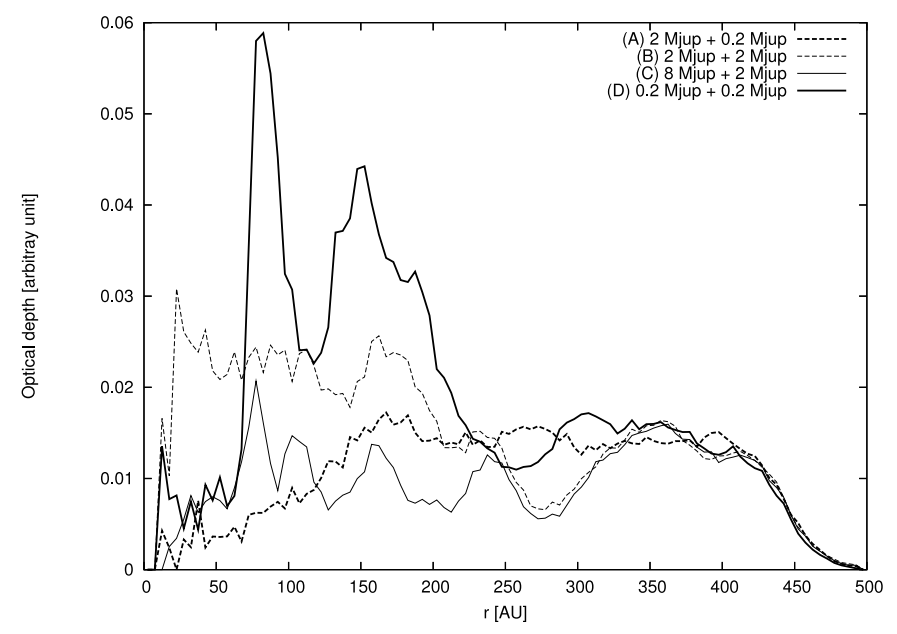

Fig. 8. Optical depth obtained by simulations with 4 different scenarios for the mass of the 2 planets, to compare to Fig. 5 .

is the one which gives the best results among the three scenarios studied in this paper.

\section{Discussion}

\subsection{Probability of the encounter}

The main drawback of the flyby scenario is that, according to our models, the binary passed to the periapsis less than 8000 years ago. The probability of observing such an event is low, making the flyby scenario statistically unlikely. On the other hand, the similar physical properties between the central star and the low mass companions suggest a common origin. The 3 stars have been independently estimated to be around $5 \mathrm{Myr}$ (Weinberger et al. 2000; Merín et al. 2004) and the differential radial velocity between the HD 141569A and the binary is only a fraction of the total speed of the primary star. But the observed radial velocities clearly show that the binary is on an escape orbit and the open spiral structure observed in the disk is also in favour of a flyby scenario, as trailing structures are created by tidal effects (Augereau et al., in prep.).

One can however reconciliate these positions with a hybrid scenario. HD 141569 could have been, in the past, a triple bound system until another star of its vicinity approached and destabilised the system, putting the binary on its current orbit. In this case, we would be observing the last periapsis passage of the binary and the consequences of a 4 star encounter. This scenario could explain the observed velocities, the common physical properties and the disk size, and it is more plausible than a sole flyby of the binary. The gravitationally bound scenario studied by Augereau \& Papaloizou (2004) and Quillen et al. (2005) can possibly be used to constraint the orbit of the binary before the encounter: typically an orbital period of several hundreds of thousand years and an apoapsis of almost 10000 AU. It appears more probable that a fourth star destabilises such an orbit that the binary randomly cross the path of the primary star at less than 1000 AU. The study by Scally \& Clarke (2001) shows that, in the Orion Nebula Cluster, almost all the stars undergo at least one encounter between 1000 and 10000 AU. On the other hand, Aarnio et al. (2008) have found that HD 141569 formed in relative isolation, tens of parsecs away from the recent sites of star formation in the Ophiucus-Scorpius-Centaurus region. The likehood of such a scenario has thus to be better estimated. Its dynamical modelling should also be investigated but this is beyond the scope of this paper. Theoretical works are however already abundant in the literature (see, for example, the series discussing many aspects of the three-body scattering from Hut \& Bahcall 1983; to Heggie et al. 1996).

\subsection{Comparison with previous works}

Other theoretical studies of the HD 141569 system can be divided in 3 groups with respect to the reproduced disk structures: the outer ring, the gap between the two rings or the extended spiral arms.

Augereau \& Papaloizou (2004) and Quillen et al. (2005) have both studied the outer ring of the disk, but using different tools: a pure $N$-body code for Augereau \& Papaloizou (2004) and a 2D hydrodynamics code for Quillen et al. (2005) assuming in the latter case that the gas and the dust are fully mixed. The main issue compared to our work is that they have considered bound orbits for the binary that are incompatible with the new measurement of radial velocities for the primary star. Interestingly, Augereau \& Papaloizou (2004) well reproduce the outer ring but not the open spiral arms structure while it is the contrary for our work. One can therefore ask if the scenario with 4 stars can reconsile these two approaches. This should also be investigated in a future paper.

Wyatt (2005) and Takeuchi \& Artymowicz (2001) have considered the opening of the gap between the two rings with very different mechanisms: a planet embedded in the disk (Wyatt 2005) or the interaction between the dust and the gas (Takeuchi $\&$ Artymowicz 2001). In the two cases, the authors do not consider the influence of the two companion stars which can be justified by our study of the kinematic constraints. It is indeed possible to obtain a binary orbit reproducing the observed radial velocities but which does not let the two $M$ stars generate any perturbation of the disk because they pass too far from the disk or because they have not yet passed at the periapsis. The planet used by Wyatt (2005) is efficient in opening the gap and generating the outer ring but, as shown in Sect. 5, it is difficult to improve this scenario in order to also explain the inner ring and the inner disk depletion. Takeuchi \& Artymowicz (2001), with an alternative scenario of dust migration due to gas friction, generate a two ring structure but the hypothesis of axisymmetry does not leave a lot of possibilities to extend this work and explain the tightly-wound spiral structure of the outer ring nor the spiral arm in the extended diffuse emission.

Ardila et al. (2005) consider several physical processes for the modelling of HD 141569: gravitation, interaction between the gas and the dust, dust generation by collisions. It is therefore, up to now, the most complete theoretical study about HD 141569. They considered the case of a flyby in order to obtain large spiral arms similar to those observed in the extended diffuse emission. They also added a $5 M_{J}$ planet, on an orbit with a semi-major axis of $100 \mathrm{AU}$ with an eccentricity of 0.6 , to explain the depleted zone below 150 AU. Altought the authors obtain good results for the the extended spiral arms and the inner hole, they did not succeed in reproducing the two rings nor the gap in between. One possible explanation is that, in our work, we obtain satisfactory results only if we let the disk evolve with the planet for several million years before the flyby. To go further in the description of the HD 141569 system, it will be interesting to use the model developed by these authors but with the initial conditions of the binary orbit and the planet parameters established by our work. 


\section{Summary and conclusion}

The binary orbit is found to be almost fixed by the observational constraint on a edge-on plane with respect to the observer. If the binary has had an influence on the disk structure, it should had a passing time at the periapsis between 5000 and 8000 years ago and a distance at periapsis between 600 and 900 AU. The scenarios with retrograde orbits better reproduce the observations but prograde orbits cannot be totally excluded. In order to reproduce the observed structures in the debris disk, it also appears that the best scenario is a flyby with 1 planet embedded in the disk. For a $2 M_{J}$ planet, its orbital eccentricity must be around 0.2 while for a $8 M_{J}$ planet, it must be below 0.1 . In the two cases, its apoapsis is about $130 \mathrm{AU}$.

This scenario is able to both reproduce the observed radial velocities of the stars and the overall structure of the disk with the model of a flyby by the binary and a embedded planet in the disk. However, this scenario is not fully satisfactory because it does not reproduce all the structures, and in particular the gap between the two annuli. Future studies could therefore extend this scenario, for example with the modelling of the gas and/or collisions, as we have not taken into account these effects on the dust dynamics.

Acknowledgements. We are grateful to Ken Marsh for the optical depth observational data and Xavier Delfosse for the discussion about the flyby encounter probability. We also thank John Papaloizou and the anonymous referee for helpful comments on this paper. Most of the computations presented were performed at the Service Commun de Calcul Intensif de l'Observatoire de Grenoble (SCCI).

\section{References}

Aarnio, A. N., Weinberger, A. J., Stassun, K. G., Mamajek, E. E., \& James, D. J. 2008, ArXiv e-prints, 809

Ardila, D. R., Lubow, S. H., Golimowski, D. A., et al. 2005, ApJ, 627, 986

Augereau, J. C., \& Papaloizou, J. C. B. 2004, A\&A, 414, 1153

Augereau, J. C., Lagrange, A. M., Mouillet, D., \& Ménard, F. 1999, A\&A, 350, L51

Beust, H. 2003, A\&A, 400, 1129

Clampin, M., Krist, J. E., Ardila, D. R., et al. 2003, AJ, 126, 385

Dent, W. R. F., Greaves, J. S., \& Coulson, I. M. 2005, MNRAS, 359, 663

Goto, M., Usuda, T., Dullemond, C. P., et al. 2006, ApJ, 652, 758

Heggie, D. C., Hut, P., \& McMillan, S. L. W. 1996, ApJ, 467, 359

Hut, P., \& Bahcall, J. N. 1983, ApJ, 268, 319

Jonkheid, B., Kamp, I., Augereau, J.-C., \& van Dishoeck, E. F. 2006, A\&A, 453, 163

Levison, H. F., \& Duncan, M. J. 1994, Icarus, 108, 18

Marsh, K. A., Silverstone, M. D., Becklin, E. E., et al. 2002, ApJ, 573, 425

Merín, B., Montesinos, B., Eiroa, C., et al. 2004, A\&A, 419, 301

Mouillet, D., Lagrange, A. M., Augereau, J. C., \& Ménard, F. 2001, A\&A, 372, L61

Pfalzner, S. 2003, ApJ, 592, 986

Quillen, A. C., Varnière, P., Minchev, I., \& Frank, A. 2005, AJ, 129, 2481

Scally, A., \& Clarke, C. 2001, MNRAS, 325, 449

Sylvester, R. J., Skinner, C. J., Barlow, M. J., \& Mannings, V. 1996, MNRAS, 279,915

Takeuchi, T., \& Artymowicz, P. 2001, ApJ, 557, 990

Thébault, P., \& Wu, Y. 2008, A\&A, 481, 713

Weinberger, A. J., Becklin, E. E., Schneider, G., et al. 1999, ApJ, 525, L53

Weinberger, A. J., Rich, R. M., Becklin, E. E., Zuckerman, B., \& Matthews, K. 2000, ApJ, 544, 937

Wisdom, J., \& Holman, M. 1991, AJ, 102, 1528

Wyatt, M. C. 2005, in BAAS, 673 